\title{
Medición de radón-222 en interiores de las viviendas de la Provincia de Lambayeque, Perú utilizando detectores LR 115
}

\author{
Indoor measurement of radon-222 in homes in the Province of Lambayeque, Peru using \\ LR 115 detectors

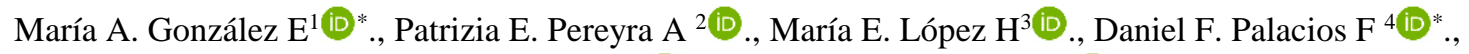 \\ César J. Guevara P. ${ }^{5 \mathbb{D}}$., y Javier A. Castañeda A ${ }^{6}$ (iD.
}

\begin{abstract}
RESUMEN
El objetivo de la presente investigación fue medir las concentraciones de radón en las viviendas de los distritos de la Provincia de Lambayeque mediante detectores LR-115 Tipo II en modo descubierto. Se utilizó detectores de trazas nucleares de estado sólido (SSNTDs), películas de nitrato de celulosa LR 115 tipo II. El número de detectores se determinó por distrito considerando la densidad poblacional y tratando de cubrir tanto la zona urbana como rural. Los detectores fueron expuestos en modo descubierto, en el interior de las viviendas durante 90 días; el procedimiento de grabado y la lectura de los detectores se realizaron en el Laboratorio de Huellas Nucleares de la PUCP. Los resultados muestran que el $15 \%$ del total de viviendas muestreadas sobrepasaron el límite máximo permisible por la normativa nacional establecida por el IPEN, encontrándose concentraciones altas de Radón en los distritos de Lambayeque, Túcume y Chóchope.
\end{abstract}

Palabras clave: radón, SSNTDs, LR 115, modo descubierto, contaminación ambiental.

\begin{abstract}
The objective of the present research was to measure radon concentrations in dwellings in the districts of the Province of Lambayeque using LR-115 Type II detectors in uncovered mode. Solid state nuclear trace detectors (SSNTDs), LR 115 Type II cellulose nitrate films, were used. The number of detectors was determined per district considering the population density and trying to cover both urban and rural areas. The detectors were exposed in uncovered mode, inside the houses for 90 days; the etching procedure and the reading of the detectors were performed at the Nuclear Fingerprint Laboratory of the PUCP. The results show that $15 \%$ of the total number of homes sampled exceeded the maximum permissible limit established by IPEN national regulations, with high concentrations of Radon found in the districts of Lambayeque, Túcume and Chóchope.
\end{abstract}

Keywords: Radon, nuclear tracks SSNTDs, LR-115, bare mode, environmental pollution.

DOI: https://doi.org/10.37787/pakamuros-unj.v9i1.163

Recibido: 22/9/2020. Aceptado: 26/12/2020

* Autor para correspondencia

1. Universidad Nacional Barranca, Av. Toribio de Luzuriaga N³76, Mz J, Barranca, Perú mgonzalez@unab.edu.pe

2. Pontificia Universidad Católica del Perú, Av. Universitaria 1801, Lima 32, Perú, ppereyr@ pucp.edu.pe

3. Pontificia Universidad Católica del Perú, Av. Universitaria 1801, Lima 32, Perú, mlopez@ pucp.edu.pe

4. Pontificia Universidad Católica del Perú, Av. Universitaria 1801, Lima 32, Perú, dpalaciosf@ pucp.edu.pe

5. Pontificia Universidad Católica del Perú, Av. Universitaria 1801, Lima 32, Perú, cesarj.guevara@pucp.edu.pe

6. Universidad Nacional Pedro Ruiz Gallo, Av. Juan XXIII 391, Lambayeque, Perú, a20024599@ pucp.edu.pe 


\section{INTRODUCCIÓN}

El radón (222Rn) proviene de la serie radiactiva natural del 238U. El radón en el ambiente se debe al contenido de 226Ra presente en los diferentes tipos de suelos. Al ser un gas se transporta desde el subsuelo hacia la atmosfera por difusión. La exhalación de radón produce que este se concentre en lugares cerrados o de poca ventilación haciéndose parte del aire que respiramos. Se caracteriza por que no es percibido por los sentidos al ser un gas noble incoloro, inodoro e insípido, hecho que hace importante su medición. La Organización Mundial de la Salud ha catalogado la exposición al gas radón como la principal causa en la incidencia de cáncer de pulmón en personas no fumadoras (OMS,2015).

Mediciones de gas radón son consideradas de importancia en la comunidad científica internacional desde principios del siglo XX, al constituir un peligro significativo para la salud humana. Por tal motivo, a nivel mundial se han realizado diversas investigaciones para medir los niveles de concentraciones de radón, debido al riesgo que conlleva su acumulación en interiores, viviendas y puestos de trabajo, siendo uno de los efectos el desarrollo de ciertos tipos de cáncer en la población. Hoy en día, hay una evidencia concreta de que el radón y su progenie pueden causar cáncer de pulmón (Efstratios et al., 2014). Por tal motivo diversos países establecieron una reglamentación definida para los niveles de concentraciones de radón en interiores.

A nivel nacional existe un Reglamento de Seguridad Radiológica que establece los niveles de concentraciones de radón en interiores (IPEN, 1997), el cual se basa en normativas dadas por la Organización Internacional de Energía Atómica. Mediciones de las concentraciones de radón a nivel nacional se han realizado con la técnica de huellas nucleares empleando detectores LR-115 tipo II en modo descubierto (Pereyra, 1998a; Pereyra et al., 2014b). Los estudios indican la presencia de radón en el aire durante las diferentes estaciones del año (Pérez, 2015), también señalan que las diferencias encontradas en las concentraciones de radón están relacionadas con el tipo de material de construcción, tamaño de las habitaciones y su ventilación (Rojas, 2016). En relación con el diseño de las viviendas, los estudios indican que en las viviendas donde el recubrimiento de las paredes es pintura, no se ha podido determinar una relación con las diferencias de los niveles de radón y el tipo de pintura; sin embargo, sí se ha podido encontrar que en algunos puntos de medición donde las paredes no presentan acabados las concentraciones superan los $200 \mathrm{~Bq} / \mathrm{m} 3$. Este resultado respalda la importancia de las capas de pintura como una buena alternativa para disminuir el radón que proviene de la exhalación de los materiales de construcción (Liza, 2017). 
En la provincia de Lambayeque no se registra ninguna investigación enfocada en determinar los niveles de concentraciones de Radón en las viviendas ni sus efectos en los habitantes, sin embargo, las estadísticas del Hospital Provincial Docente "Belén" de la ciudad de Lambayeque registran una alta tasa de incidencia de cáncer pulmonar para esta provincia, esto alcanza una notoria relevancia dadas las características especiales de las viviendas en la región

En este estudio se pretende estimar las concentraciones de radón en las viviendas de los distritos de la Provincia de Lambayeque mediante detectores LR-115 Tipo II en modo descubierto, estableciendo una relación con el material de construcción y el tipo del recubrimiento del suelo de las casas de la zona de estudio.

\section{MATERIALES Y MÉTODOS}

La población de muestreo fueron las viviendas de la provincia de Lambayeque localizada en el norte del Perú, está conformada por 12 distritos y tiene una densidad poblacional de $32 \mathrm{hab} . / \mathrm{km}^{2}$. Los criterios para determinar el número de viviendas a monitorear fueron establecidos en el Proyecto 120-PNCIPPIAP-2015, basados en la densidad poblacional y presentados en las tablas 1 y 2.

Tabla 1. Consideraciones de muestreo

\begin{tabular}{lcc}
\hline Criterio & habs./km & casas/km $\mathbf{k}^{\mathbf{2}}$ \\
\hline TIPO A & 300 a 450 & 8 \\
TIPO B & De 150 a 300 & 4 \\
TIPOC & De 0 a 150 & 2 \\
\hline
\end{tabular}

Tabla 2. Tipo de recubrimiento de las viviendas

\begin{tabular}{cc}
\hline Criterio & Tipo de recubrimiento de la vivienda \\
\hline TIPO A & Falso piso o contrapiso, piso de tierra, cemento pulido y mayólica \\
\hline TIPO B & Mayólica, piso de tierra, cemento pulido \\
\hline TIPO C & Piso de tierra, falso piso, cemento pulido \\
\hline
\end{tabular}




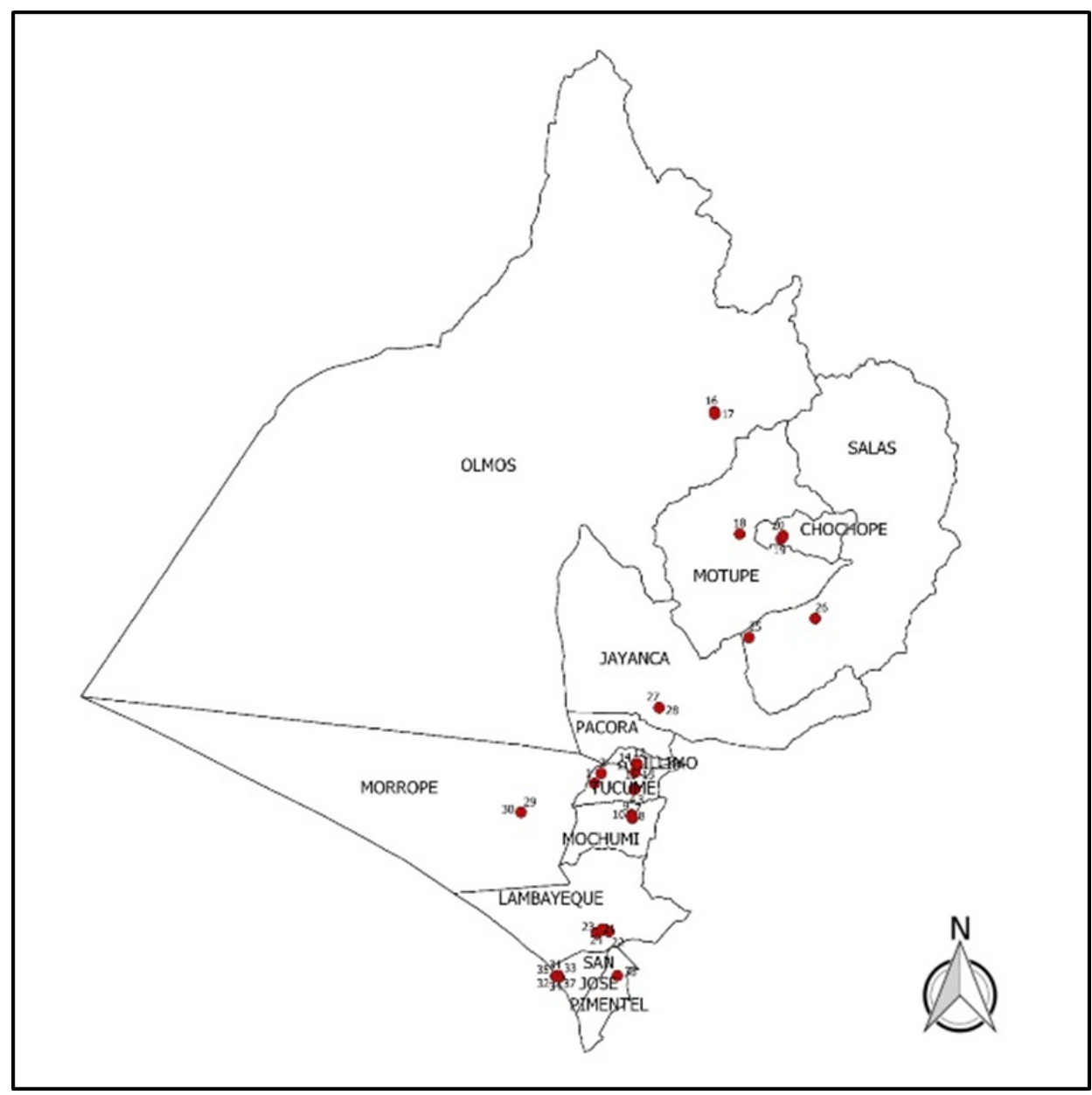

Figura 1. Puntos de medición en la provincia de Lambayeque, Perú

Figura 1, se muestra un total de 46 viviendas a monitorear, los cuales son los puntos de medición. Se expusieron detectores LR-115 tipo II de 2 x $2 \mathrm{~cm}$ de tamaño en modo descubierto, los cuales fueron colocados en las paredes de ambientes en el primer piso de las viviendas. Las condiciones ambientales en promedio fueron de $23^{\circ} \mathrm{C}$ de temperatura y $69 \%$ de humedad promedio. Las trazas latentes (a escala nanométrica) causada por el impacto de partículas alfa son amplificadas mediante un proceso de grabado utilizando una solución de $\mathrm{NaOH}$ a $2.5 \mathrm{~N}$ a una temperatura de $60 \pm 5^{\circ} \mathrm{C}$ durante 90 minutos (Ramsiya et al., 2017), posteriormente se realizó un proceso de enjuague y secado.

Las trazas reveladas generadas fueron observadas con un microscopio óptico. El error debido a la lectura y el número de campos leídos en los detectores es menor al $20 \%$.

La densidad efectiva de trazas $\left(\right.$ trazas $/ \mathrm{cm}^{2}$ ) de los detectores está dada por (1) 


$$
\rho_{e f}=\rho_{T}-\rho_{f}
$$

Donde $\rho_{T}$ es la densidad total de trazas de los detectores y $\rho_{f}$ es la densidad de trazas de fondo que corresponde a los detectores que no han sido expuestos.

Según Ferrer (2003), (la concentración de radón $C_{222 R n}\left(\mathrm{~Bq} / \mathrm{m}^{3}\right)$ se calcula mediante la ecuación (2)

$$
C_{222 R n}=\frac{\rho_{e f}}{K \times t_{\text {exp }}}
$$

Donde $t_{\text {exp }}$ es el tiempo de exposición en días (90 días) y $K$ es el factor de calibración ( $0.020 \pm 0.002$ trazas $\mathrm{cm}^{-2} / \mathrm{Bq}$ días $\mathrm{m}^{-3}$ ) determinado exponiendo detectores dentro de una cámara de radón.

\section{RESULTADOS}

Los resultados de las densidades de trazas de los detectores y la concentración de radón $\left({ }^{222} \mathrm{Rn}\right)$ de 39 viviendas monitoreadas son presentan en la Tabla 3 con su respectiva codificación.

Tabla 3: Resultados de la densidad de trazas y promedio de la concentración de radón del ambiente monitoreado

\begin{tabular}{lcc}
\hline Código & $\begin{array}{c}\boldsymbol{\rho}_{\boldsymbol{e f}} \\
\left(\mathbf{T r} \cdot \mathbf{m m}^{\mathbf{2}}\right)\end{array}$ & $\begin{array}{c}\boldsymbol{C}_{\mathbf{2 2 2 n} \boldsymbol{n}} \\
\left(\mathbf{B q} / \mathbf{m}^{\mathbf{3}}\right)\end{array}$ \\
\hline D-T3-A & $5.33 \pm 0.41$ & $211 \pm 16$ \\
D-T3-B & $2.29 \pm 0.28$ & $91 \pm 11$ \\
D-T3-C & $2.92 \pm 0.29$ & $116 \pm 12$ \\
D-T3-E & $2.43 \pm 0.35$ & $96 \pm 14$ \\
D-T3-F & $7.92 \pm 0.63$ & $314 \pm 25$ \\
D-T3-G & $1.18 \pm 0.23$ & $47 \pm 9$ \\
D-M5-A & $4.55 \pm 0.43$ & $181 \pm 17$ \\
D-M5-B & $2.00 \pm 0.30$ & $80 \pm 12$ \\
D-M5-C & $1.72 \pm 0.27$ & $68 \pm 11$ \\
D-M5-D & $2.10 \pm 0.25$ & $83 \pm 10$ \\
D-I1-C & $0.26 \pm 0.09$ & $15 \pm 4$ \\
D-I1-D & $0.21 \pm 0.14$ & $15 \pm 5$ \\
D-I1-E & $2.74 \pm 0.33$ & $109 \pm 13$ \\
D-I1-G & $0.12 \pm 0,09$ & $15 \pm 4$ \\
D-I1.H & $0.40 \pm 0.17$ & $16 \pm 7$ \\
D-P11-B & $0.31 \pm 0.10$ & $15 \pm 4$ \\
D-OLM6-A & $2.48 \pm 0.30$ & $98 \pm 12$ \\
D-OLM6-B & $1.51 \pm 0.22$ & $60 \pm 9$ \\
D-MT10-A & $2.38 \pm 0.29$ & $94 \pm 12$ \\
D-MT10-B & $1.51 \pm 0.20$ & $60 \pm 8$ \\
D-CH7-A & $6.55 \pm 0.57$ & $260 \pm 22$ \\
\hline
\end{tabular}




\begin{tabular}{lcc}
\hline D-CH7-B & $6.15 \pm 0.68$ & $244 \pm 27$ \\
D-L4-A & $3.00 \pm 0.26$ & $119 \pm 10$ \\
D-L4-B & $9.24 \pm 0.82$ & $367 \pm 32$ \\
D-L4-C & $1.75 \pm 0,31$ & $69 \pm 12$ \\
D-L4-D & $3.14 \pm 0.46$ & $124 \pm 18$ \\
D-SL12-A & $2.64 \pm 0.41$ & $105 \pm 16$ \\
D-SL12-B & $0.12 \pm 0.08$ & $15 \pm 3$ \\
D-J8-A & $2.90 \pm 0.37$ & $115 \pm 15$ \\
D-J8-B & $5.19 \pm 0.43$ & $205 \pm 17$ \\
D-MP9-A & $1.11 \pm 0.18$ & $44 \pm 7$ \\
D-MP9-B & $0.21 \pm 0.09$ & $15 \pm 3$ \\
D-SJ2-A & $2.01 \pm 0.42$ & $80 \pm 16$ \\
D-SJ2-B & $2.64 \pm 0.36$ & $105 \pm 14$ \\
D-SJ2-C & $2.83 \pm 0.21$ & $112 \pm 8$ \\
D-SJ2-D & $2.57 \pm 0.31$ & $102 \pm 12$ \\
D-SJ2-E & $0.90 \pm 0.17$ & $36 \pm 7$ \\
D-SJ2-F & $2.05 \pm 0.28$ & $81 \pm 11$ \\
D-SJ2-G & $1.20 \pm 0.27$ & $48 \pm 10$ \\
\hline
\end{tabular}

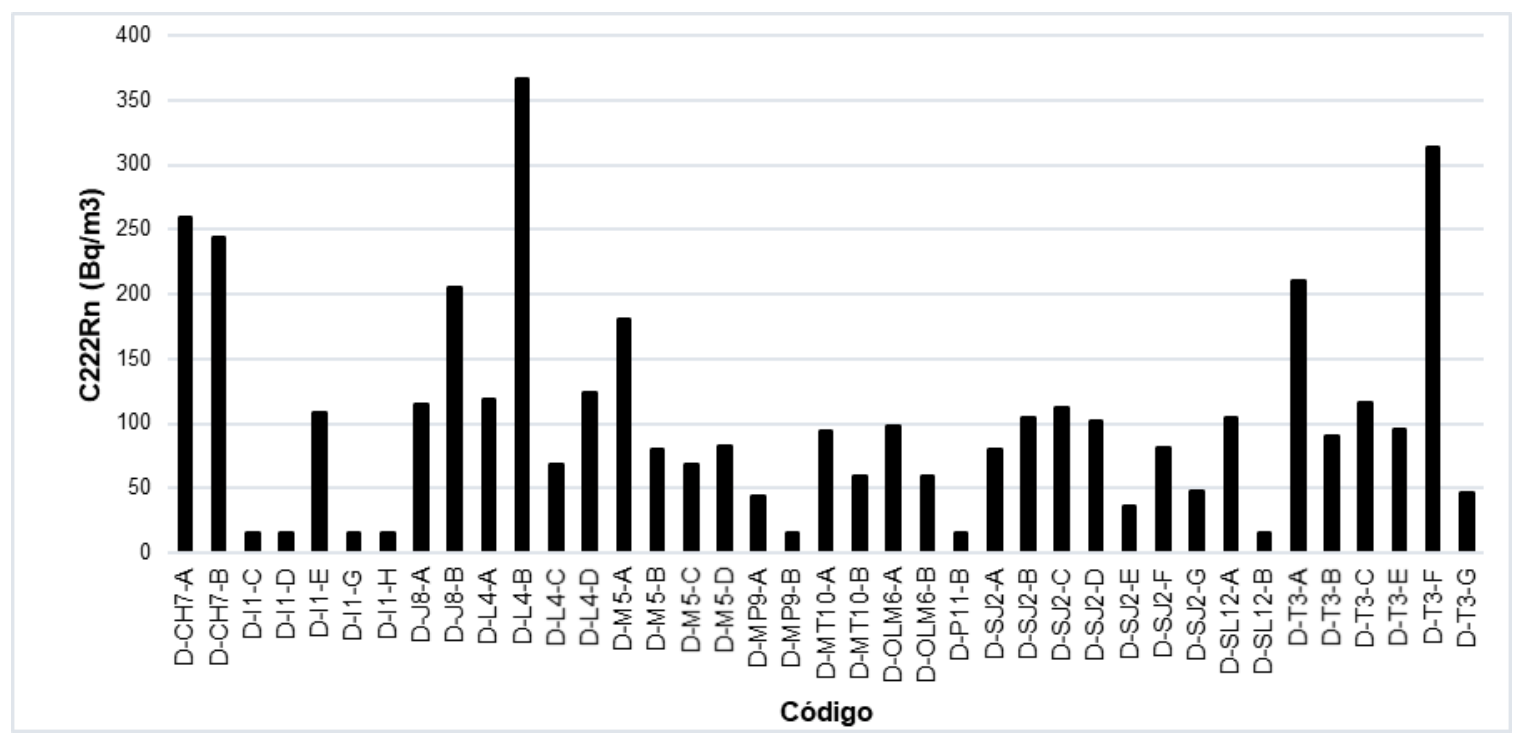

Figura 2: Concentraciones de radón en las viviendas de Lambayeque, Perú

Las concentraciones promedio de radón obtenidas se presentan en la Figura 2. El valor máximo obtenido es de $367 \pm 32 \mathrm{~Bq} / \mathrm{m}^{3}$, el valor mínimo es de $15 \pm 3 \mathrm{~Bq} / \mathrm{m}^{3}$ y el valor promedio de $103.3 \pm 2.2 \mathrm{~Bq} / \mathrm{m}^{3}$. Las concentraciones de radón en promedio se presentan mediante un histograma (Figura 3). 


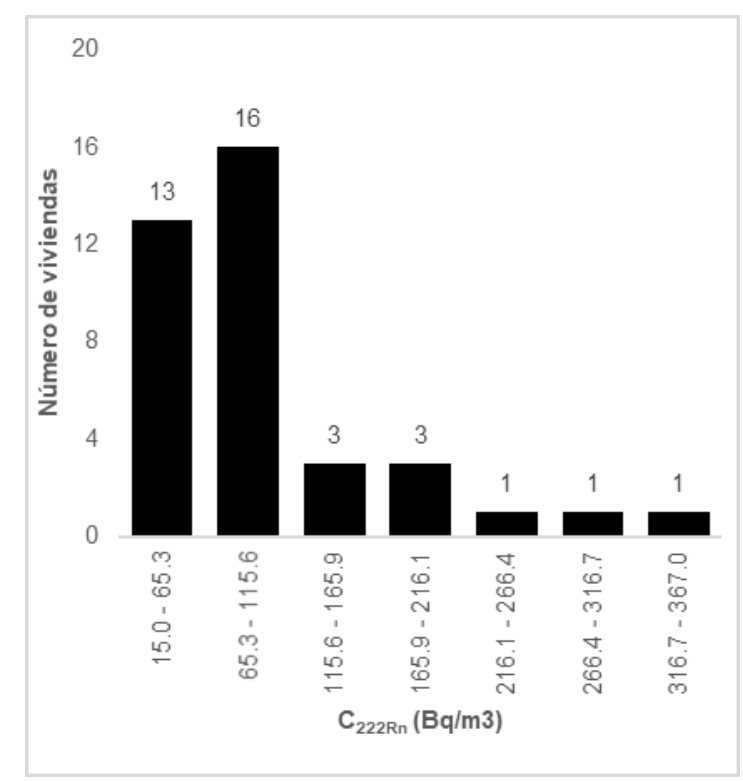

Figura 3: Histograma de las concentraciones promedio de radón

\section{DISCUSIÓN}

La distribución de frecuencias (número de viviendas) de las concentraciones de radón medidas en los ambientes se representan en la figura 4, al parecer siguen una distribución log-normal (Liza, 2017; Ramsiya, 2017).

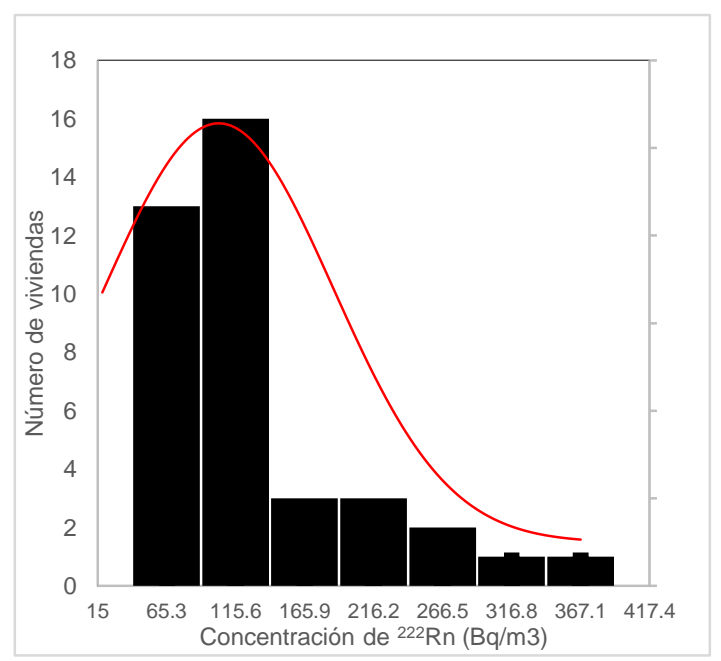

Figura 4. Histograma de frecuencias de las concentraciones de radón

Esta hipótesis puede ser verificada realizando un histograma de frecuencias del logaritmo natural (ln) de las concentraciones de radón y evaluar su normalidad mediante el Test de Anderson Darling.

En la Figura 5, el histograma de frecuencias del $\ln C_{222 R n}$ presenta un ancho de clase de $\ln C_{222 R n}=0.4$, una frecuencia media de $13 \mathrm{y}$ un valor de clase media de $\ln C_{222 R n}=4.8\left(C_{222 R n}=116 \mathrm{~Bq} / \mathrm{m}^{3}\right)$. 


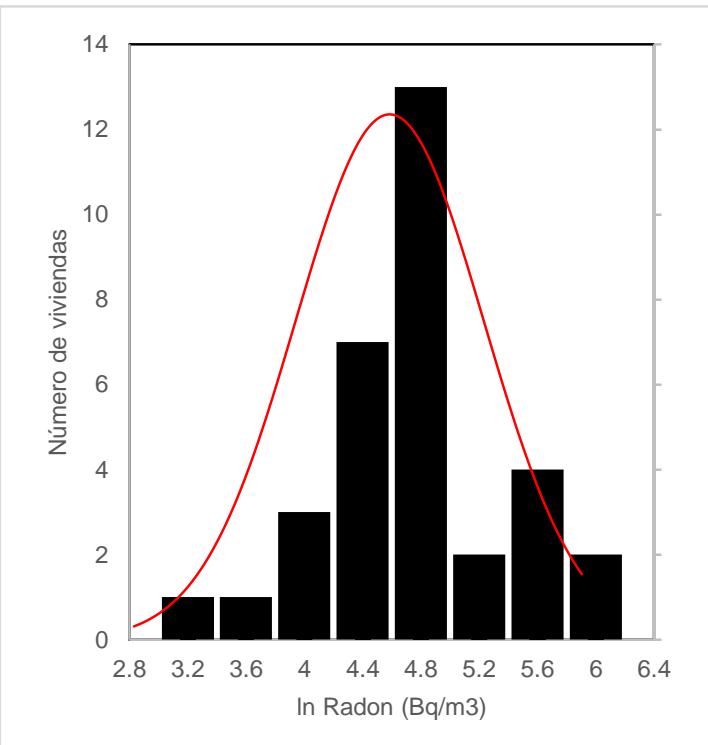

Figura 5. Histograma de frecuencias del logaritmo natural de las concentraciones radón (pág. 37- 42. [8]).

Al trazar los datos contra una distribución normal teórica, estos deben formar aproximadamente una línea recta, las salidas de la línea recta indican las desviaciones de la normalidad. El test de Anderson Darling representado en la Figura 6 determina un P-valor $=0.165$.

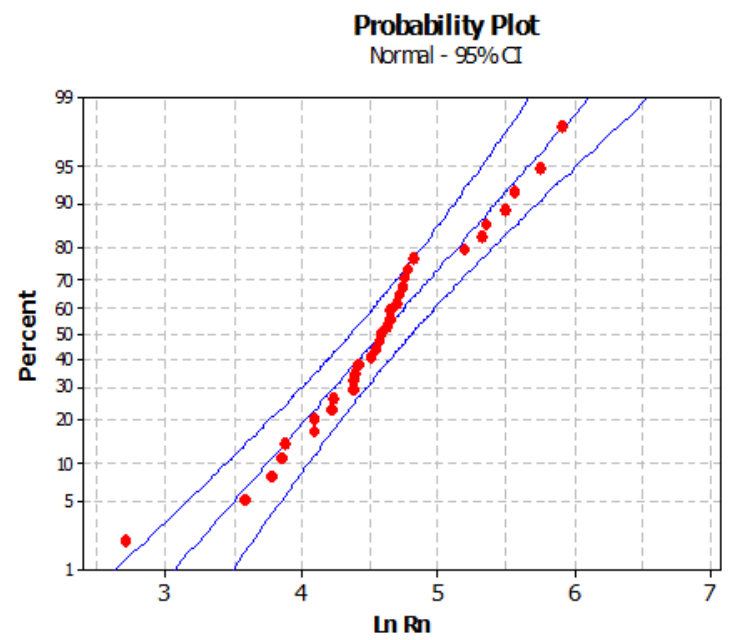

Figura 6. Test de Anderson Darling del periodo de monitoreo

\section{CONCLUSIONES}

De acuerdo con los resultados el $15 \%$ de las viviendas superan los $200 \mathrm{~Bq} / \mathrm{m}^{3}$ de concentración de radón $\left({ }^{222} \mathrm{Rn}\right)$, encontrándose en los distritos de Lambayeque, Túcume y Chóchope concentraciones de $367 \pm$ $32 \mathrm{~Bq} / \mathrm{m}^{3}, 314 \pm 25 \mathrm{~Bq} / \mathrm{m}^{3}$ y $260 \pm 22 \mathrm{~Bq} / \mathrm{m}^{3}$ de radón respectivamente. Estos lugares de medición se 
diferenciaron con los otros en aspectos como, la antigüedad de construcción, material de construcción utilizado, tipo de piso y además por ser ambientes de muy poca ventilación.

De acuerdo con el Test de Anderson Darling el P-valor $(0,165)$ es mayor a 0.05 (nivel de confianza del $95 \%)$. Por lo tanto, se acepta la hipótesis nula que la distribución de las concentraciones de radón encontradas en las viviendas se ajusta a una distribución log normal. Los resultados de estudio permitieron realizar el primer mapa de radón de la Provincia de Lambayeque representado en la figura 6.

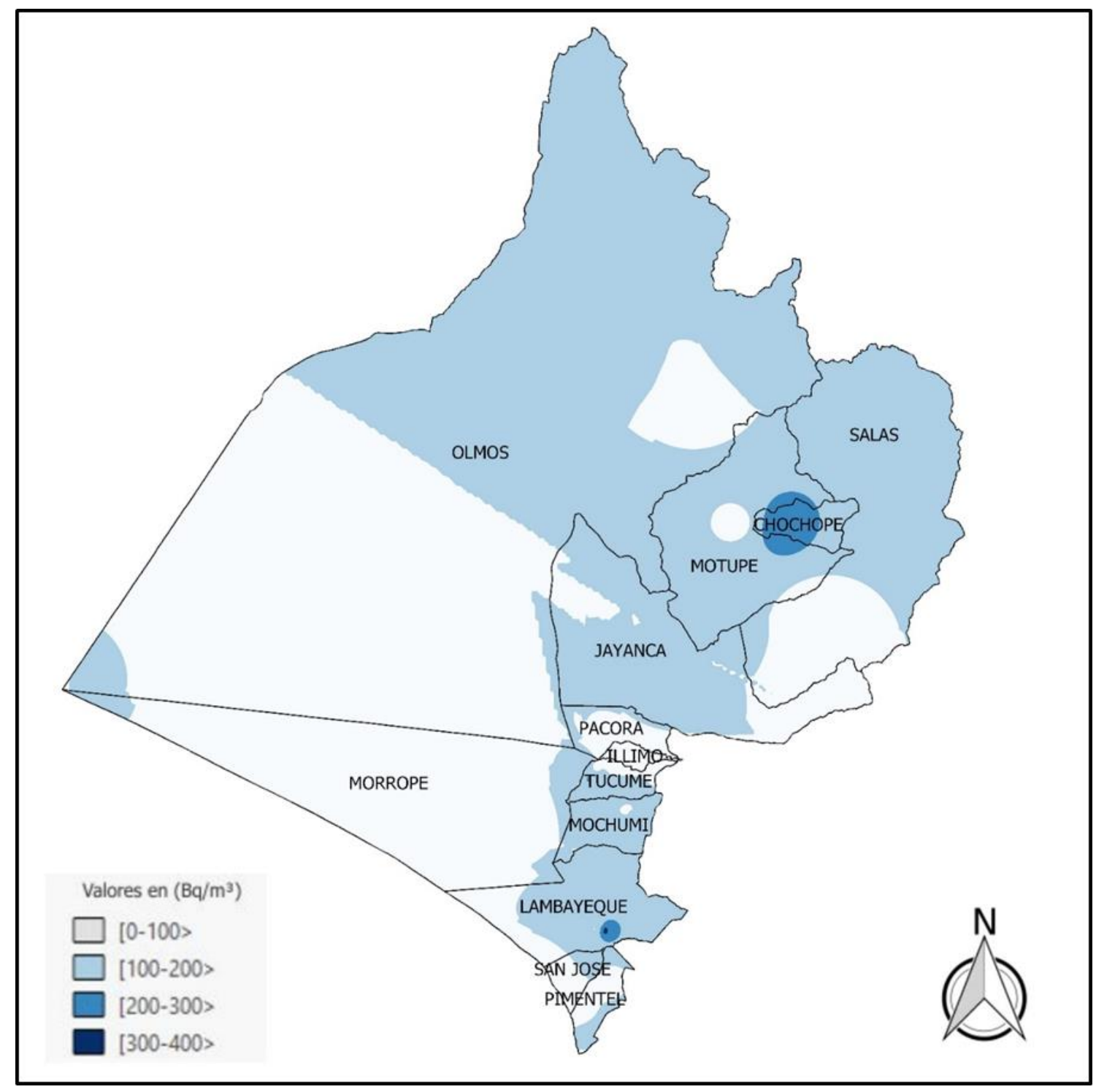

Figura 6. Mapa de radón de la provincia de Lambayeque, Perú 


\section{AGRADECIMIENTOS}

A todas las familias que colaboraron en esta investigación, permitiendo la instalación de los detectores en sus viviendas. A los miembros del Grupo de Investigación en Técnicas de Huellas Nucleares GITHUNU-PUCP y al Instituto de Energía Nuclear- IPEN, por proporcionar los detectores, por su asesoría y haber brindado su laboratorio.

\section{REFERENCIAS BIBLIOGRÁFICAS}

OMS. 2015. "Una perspectiva de salud pública". https://www.who.int/phe/publications/indoor_randon_handbook/es/

Efstratios, G. Vogiannis and Dimitrios, N. (2014). Radon Sources and Associated Risk in Terms of Exposure and Dose. Front Public Health. 2:207. doi: 10.3389/fpubh.2014.00207

Instituto Peruano de Energía Nuclear IPEN. (1997). Reglamento de seguridad radiológica. https://www.ipen.gob.pe/radon/radon.html

Pereyra, P. (1998 a). Detección de Rn222 en ambientes interiores utilizando la técnica de Huellas nucleares. (Tesis de Licenciatura). Pontificia Universidad Católica del Perú. Lima.

Pereyra, P., López, M y Pérez, B. (2014b). Mediciones de la Concentración de Radón 222 en residencias de Lima, Perú. http://hdl.handle.net/20.500.12404/6724

Pérez, B. (2015). Estudio de la contaminación ambiental debido a elementos radiactivos naturales. (Tesis de bachiller). Pontificia Universidad Católica del Perú. Lima.

Rojas, J. (2016). Monitoreo de Radón en la zona sur de Lima. (Tesis de Maestría). Pontificia Universidad Católica del Perú. Lima. http://hdl.handle.net/20.500.12404/7441

Liza, R. (2017). Mapeo de Niveles de radón 222 en el Distrito de San Martin de Porres (Lima - Perú). (Tesis de Maestría). Pontificia Universidad Católica del Perú. Lima. http://hdl.handle.net/20.500.12404/8656

Ramsiya M., et al. Estimation of indoor radón and thoron in dwellings of Palakkas, Kerala, India using solid state nuclear track detectors, Journal of Radiation Research and Applied Sciences, 2017, vol.10, no 3, p. 269-272. https://doi.org/10.1016/j.jrras.2017.05.004

Eappen, K.P, Mayya, Y.S. “Calibration factors for LR 115 (type 2) based radón thoron discriminating dosimeters", Radiation Measurements, 38, 5-17 (2004). DOI 10.1016/j.radmeas.2003.09.003

Ferrer, A. (2003). Física Nuclear y de Partículas. Valencia, España. QUILES Artes Gráficas S. A.Universitat. ISBN 8437065682, 9788437065687 
Fleischer, R., Buford, P.; Walker, R., (1975). Nuclear Tracks in Solids: Principles and Applications. Los Angeles, London. The Regents the University of California Press. Bibcode: 1975ucb..book.....F

Kodalpha Radon- Dosimeters and LR 115 SSNTD Specifications. Recuperado de www.gtanalytic.at/downloads_en/Kod_tech_det.pdf

Lantz, P., Mendez, D; Philbert M. (2013). Radon, smoking, and lung cáncer: the need to refocus radón control policy. US National Library of Medicine National Institutes of Health. doi: 10.2105/AJPH.2012.300926 